\title{
Author Correction: Plasmon-induced trap filling at grain boundaries in perovskite solar cells
}

Kai Yao De, Siqi Li, Zhiliang Liu, Yiran Ying De, Petr Dvořák, Linfeng Fei@D, Tomáš Šikola, Haitao Huang,

Peter Nordlander (D, Alex K-Y. Jen (1D) and Dangyuan Lei (D)

Correction to: Light: Science \& Applications

https://doi.org/10.1038/s41377-021-00662-y, published online 28 October 2021

In the version of this article initially published, there were errors in Fig. 3a. The PL mapping image of Target sample (Au@PAT-modified $\mathrm{MAPbI}_{3}$ ) after treatment was incorrectly positioned on the Control sample (PAT-treated $\mathrm{MAPbI}_{3}$ ). In addition, the color scales were drawn in the wrong orientation.
So we would like to correct Fig. 3 in the initial published version to the following figure:

We would like to apologize for any inconvenience this may have caused.

The original article has been updated.

Published online: 18 January 2022 


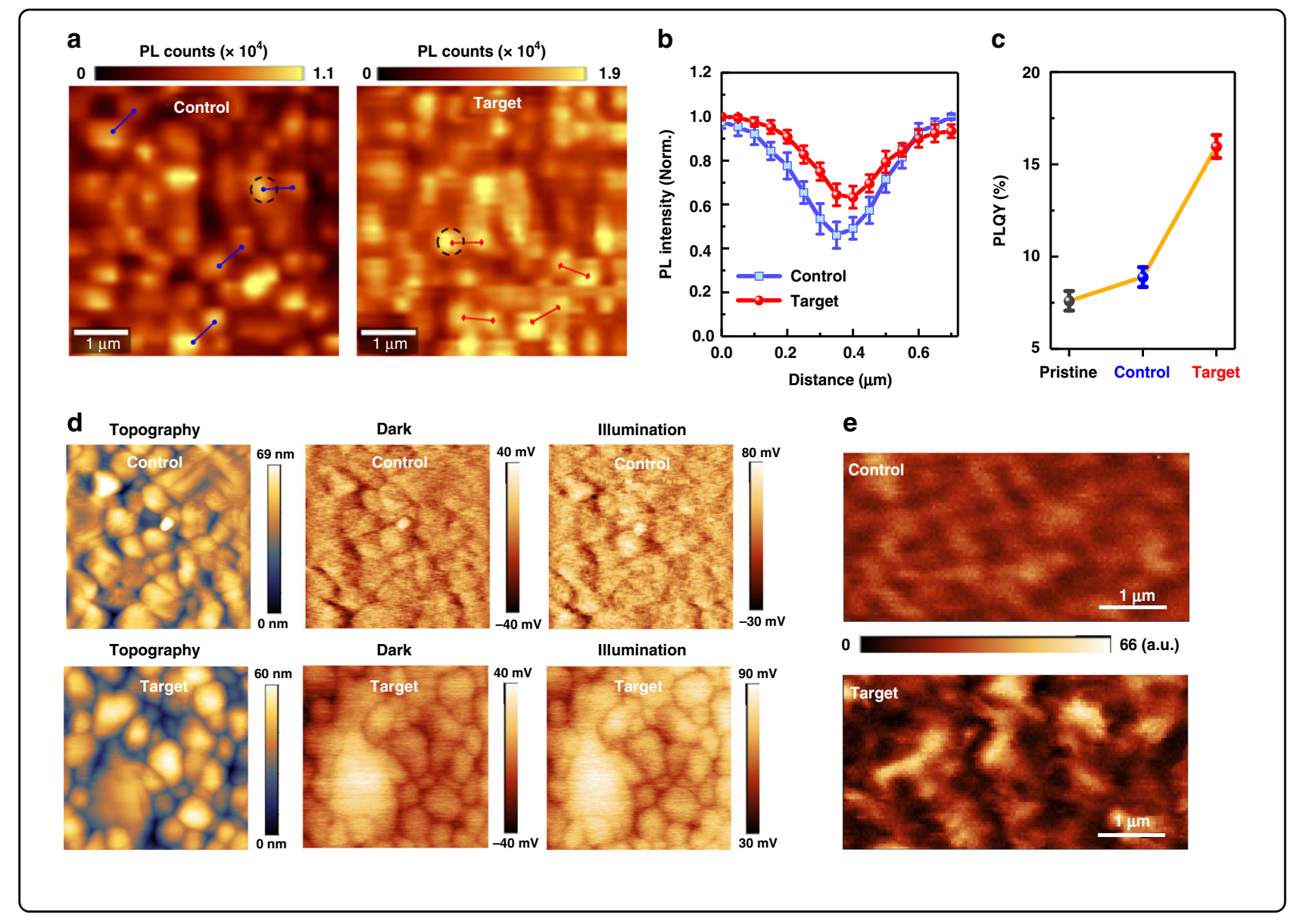

